\title{
APPLICATION OF OMNI-DIRECTIONAL TEXTURE ANALYSIS TO SAR IMAGES FOR LEVEE LANDSLIDE DETECTION
}

\author{
Matthew A. Lee ${ }^{1}$, James V. Aanstoos ${ }^{2}$, Lori Mann Bruce ${ }^{2}$, and Saurabh Prasad ${ }^{3}$ \\ 1. Graduate Student, Mississippi State University, MS, USA \\ 2. Professor, Mississippi State University, MS, USA \\ 3. Professor, University of Houston, Houston, TX, USA
}

\begin{abstract}
This paper explores different types of gray level co-occurrence matrix (GLCM) [2] texture features for automated detection of landslides on levees using remotely sensed Synthetic Aperture Radar (SAR). Two approaches of texture analysis are investigated: one based on a rubber band straightening transform (RBST) which has been used extensively in the past in the medical imaging community, and one based on the authors' developed approach of spiral straightening transform (SST). The transforms are used to project a circular region in the image to a rectangular representation where texture feature extraction can be applied. Straightforward linear discriminant analysis, for feature reduction and optimization, and maximum likelihood methods, for classification, are also utilized. The proposed system was tested on L-band SAR data with $\mathrm{HH}, \mathrm{HV}$, and VV polarizations collected from NASA's UAVSAR of the Mississippi River levee system between Vicksburg, MS and Clarksdale, MS, USA. The proposed approach is shown to detect all known levee landslides in the test area with a low number of false positives.
\end{abstract}

\section{INTRODUCTION}

Earthen Levees protect large areas of populated and cultivated land in the United States from flooding. In the United States there are more than 150,000 kilometers of levee structures of varying designs and conditions. The potential loss of life and property associated with the catastrophic failure of levees can be extremely large [1]. Currently, there are limited processes in place to prioritize the monitoring of large numbers of dam and levee structures. There is a need to prioritize the monitoring of the network of dam and levee structures. Levee managers and federal agencies will benefit from any tools allowing them to assess levee health rapidly with robust techniques that identify, classify and prioritize levee vulnerabilities with lower costs than traditional programs not based on the use of remote sensing. This paper explores different types of gray level co-occurrence matrix (GLCM) [2] texture features which can be used to identify landslides on levees using Synthetic Aperture Radar (SAR).

\section{DATA}

This experiment used L-band SAR data collected from JPL's UAVSAR [3] of the Mississippi River levee system between Vicksburg, MS and Clarksdale, MS. Each pixel has a spatial resolution of $4 \mathrm{~m} \times 4 \mathrm{~m}$. The instrument created separate backscatter intensity images for three different polarizations. These polarizations are $\mathrm{HH}, \mathrm{HV}$, and $\mathrm{VV}$. The aircraft made two passes over the collection area. In the first pass, the plane traveled south to north with the sensor looking westward, and in the second pass, the plane traveled north to south with the sensor looking eastward.

The data was collected on June 16, 2009, which according to the National Ocean and Atmospheric Administration (NOAA) data was hotter than usual and was the $6^{\text {th }}$ driest June since 1895 for that region [4]. Ground truth for landslides on the levees was obtained using optical imagery from the National Agriculture Imagery Program (NAIP), the Army Core of Engineers records, and this
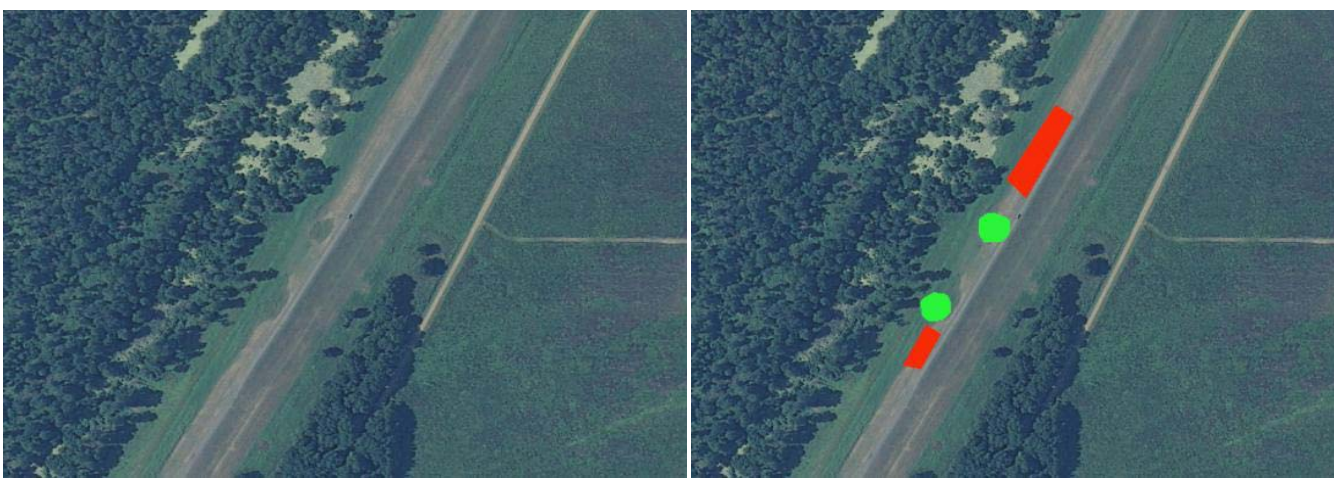

Figure 1. Left Image: Actual levee segment. Right Image: Mask segment. Green: landslide; Red: healthy levee. 


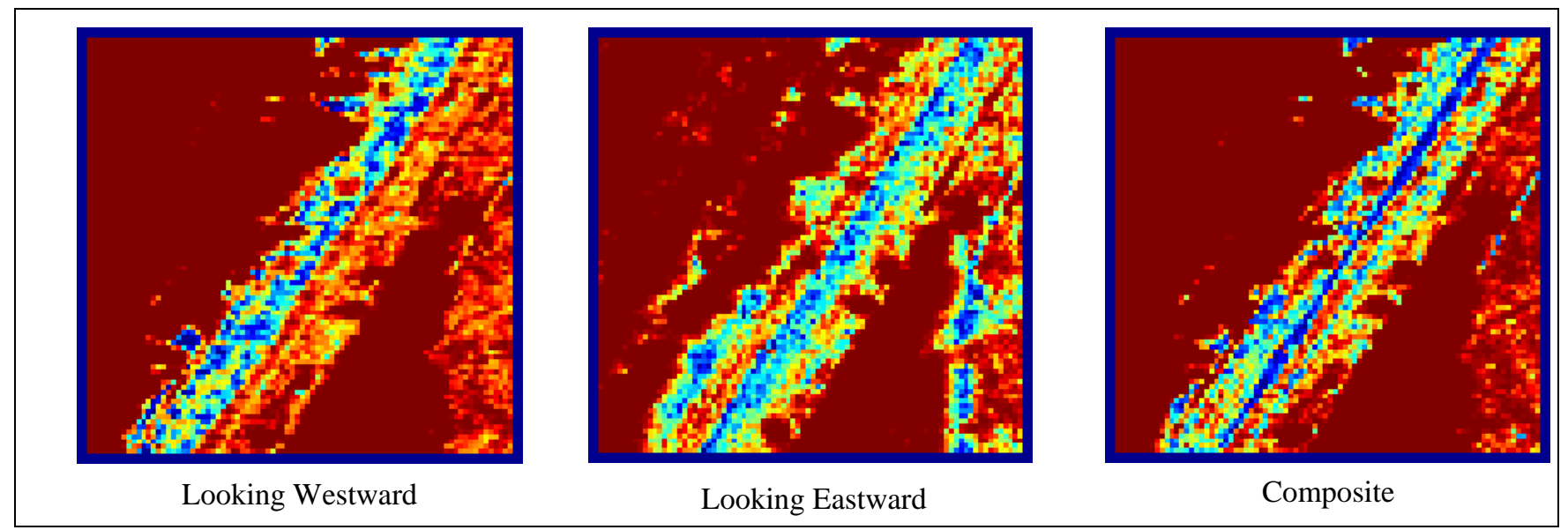

Figure 2. SAR images of the levee.

study's researchers manually observing the levees. The landslides studied in this experiment were confirmed by at least two of these sources.

\section{METHODOLOGIES}

For detecting landslides on the levees, we developed a supervised classification system that fuses texture information from the $\mathrm{HH}$, $\mathrm{HV}$, and VV polarizations for both passes. There are four main steps to the classification system. They are (1) compute a uniformly illuminated composite image from the six input images (HV, HV, and VV for both passes); (2) extract spatial features from the six input images plus the composite image; (3) select features and train; and (4) classify the levee section using maximum likelihood classification. The following subsections detail each of these steps.

\subsection{Computing the composite image}

One of the disadvantages of using SAR imagery for levee applications is that the image tends to be dim or in shadow on the reverse slopes of hills. Since an earthen levee is essentially a hill extended in a direction that parallels the river's course, it is difficult to collect imagery of both sides of a levee using a single pass with an airborne SAR since one side will be darker than the other. This was overcome by using data from two passes with the sensor looking in opposite directions at the target area to create a composite image. The first step in computing the composite image is to reduce the images from all the polarizations into a single grayscale image for each pass. This is done by computing the Euclidean distance of the vector $\left\langle H H_{x, y}, H V_{x, y}, V V_{x, y}\right\rangle$ for each pixel $(x, y)$. Next, both of the grayscale images are normalized by subtracting the mean for each image and then dividing by the standard deviation. This step ensures that both images have the same intensity. Finally, the grayscale images are combined by selecting the maximum value for each pixel. Figure 2 shows the
$\mathrm{HH}$ images for both passes and the composite for a subset of the levee section.

\subsection{Omni-Directional Texture Analysis}

The omni-directional texture analysis used in this study is based on GLCM texture analysis. In standard GLCM texture analysis, a rectangular region is segmented, and a co-occurrence matrix is constructed by shifting the position operator across the rows of the region. Then, features are computed from the co-occurrence matrix. This technique is useful when there are textures that have a pattern in a particular direction that corresponds to the position operator. However, if there is no linear pattern to the texture, or the pattern is not always in the same direction, the standard technique may not be as effective. In the omni-directional texture analysis in this paper, a circular region instead of a rectangular region is segmented. The circular region is then re-sampled to create a rectangular image, and a position operator is then used to create a co-occurrence matrix. This paper investigates two different resampling patterns. The first is based on the Rubber Band Straightening Transform (RBST) [5]; since the regions are always a circle, the re-sampling pattern resembles radial spokes. RBST has been used extensively in automated mammographic image analysis, where texture features are extracted from a banded region around the perimeter of a mass. However, the RBST method assumes the existence of an object with a distinct perimeter that can be segmented. It also assumes there is no interest in features extracted from the interior of the segmented object. The authors have developed a new approach. The newly developed pattern samples the region by following an evenly spaced spiral path from the center of the region to the outside edge. This sampling pattern is called the Spiral Straightening Transform (SST) [6]. For each of the RBST and the SST approaches, GLCM texture analysis is conducted on the re-sampled images to produce a very large set of texture features. 


\subsection{Feature Selection}

Features are selected using stepwise linear discriminate analysis (SLDA), which included both a forward selection and backward rejection phase. Prior to the forward selection phase, features are sorted by Bhattacharyya distance, so the highest ranked features are included first.

\subsection{Classifier}

In this study, a maximum likelihood classifier with linear discriminant analysis [2] was used. The accuracy was measured using two different methods. The first was to compute a standard pixel-by-pixel confusion matrix using the ground truth and the classification map output from the classifier. While being a standard approach, a confusion matrix does not account for spatial distribution of the classes in the classification map, and since landslides typically are larger than a single pixel, it is only necessary to detect a subset of the landslide pixels. Thus, for the second metric, contiguous regions classified as landslides are segmented and determined to be true positives if the region corresponds to an actual landslide. A region corresponds to a landslide if the center of the region is within a specified distance tolerance of the landslide. The tolerance is determined by the georegistration accuracy of the SAR image, which is not constant because of foreshortening.

\subsection{Training and Testing}

Typically, pixels in SAR images possess a high degree of spatial correlation. Thus, randomly selecting pixels for training and testing from the same levee section can lead to deceiving conclusions about the accuracy of a technique since highly correlated, adjacent pixels may be selected for both training and testing. In order to avoid this, pixels for training and testing were always selected from different sections of the levee in a leave-one-out fashion with one section used for testing and the other levee sections used for training the classifier. The three sections of levee used in this study were each separated by several kilometers with one on a different side of the river. Thus, spatial correlation between the training and testing pixels was negligible.

\section{RESULTS}

In this study, the classification accuracy was determined using only standard GLCM, using only omni-directional GLCM, and using both types of features together. The confusion matrices for these three tests are shown in the following tables. Judging by the confusion matrices and pixel-by-pixel classification accuracy, there is little difference between the three approaches. However, when the spatial distribution of the classification map is considered, it becomes clear that the technique that uses both types of GLCM features detects all four landslides and has fewer false positive regions (see Table 4).

\section{CONCLUSIONS}

From the results, a general trend can be seen where standard GLCM produces a classifier that is more likely to reject (thus producing fewer true positives and fewer false positives), and omni-directional GLCM is more likely to accept (thus producing more true positives and false positives). When using both types of features, the classifier results are balanced between using one type of features or the other. This allows the classifier to detect all of the landslides while producing fewer false positives than using omni-directional GLCM only. One would expect that standard GLCM would be more selective because of its more directionally selective sampling and position operator, and thus have fewer false positives and true positives. The omni-directional GLCM is less selective, which helps it detect the landslides better but makes it more likely confused by similar textures that have a different directional element. In the levee experiment, the direction of the levees only varied by about 45 degrees, so the directional components to the texture likely varied by about 45 degrees also. One would expect omni-directional GLCM to be most advantageous in cases where the directional components of the target texture vary by a full 360 degree range and when detecting all targets is more important than producing false positives. There are a great number of applications that fall in this category in geosciences and remote sensing.

Table 1. Confusion Matrix using standard GLCM only.

\begin{tabular}{|l|r|r|r|}
\hline & slide & normal & $\begin{array}{r}\text { Producer } \\
\text { Accuracy }\end{array}$ \\
\hline Slide & 38 & 83 & 0.31 \\
\hline normal & 73 & 3375 & 0.98 \\
\hline $\begin{array}{l}\text { User } \\
\text { Accuracy }\end{array}$ & 0.34 & 0.98 & 0.96 \\
\hline
\end{tabular}

Table 2. Confusion Matrix using omni-directional GLCM only.

\begin{tabular}{|l|r|r|r|}
\hline & slide & Normal & $\begin{array}{r}\text { Producer } \\
\text { Accuracy }\end{array}$ \\
\hline slide & 26 & 95 & 0.21 \\
\hline normal & 111 & 3337 & 0.97 \\
\hline $\begin{array}{l}\text { User } \\
\text { Accuracy }\end{array}$ & 0.19 & 0.97 & 0.94 \\
\hline
\end{tabular}


Table 3. Confusion Matrix using both types of GLCM

\begin{tabular}{|l|r|r|r|}
\hline & slide & normal & $\begin{array}{r}\text { Producer } \\
\text { Accuracy }\end{array}$ \\
\hline slide & 51 & 70 & 0.42 \\
\hline normal & 146 & 3302 & 0.96 \\
\hline $\begin{array}{l}\text { User } \\
\text { Accuracy }\end{array}$ & 0.26 & 0.98 & 0.94 \\
\hline
\end{tabular}

Table 4. True positives and false positives for all three techniques. Note that there are 4 landslides, so a maximum of 4 true positives.

\begin{tabular}{|l|r|r|}
\hline & true positive & false positive \\
\hline Standard GLCM & 2 & 13 \\
\hline Omni-Directional GLCM & 4 & 36 \\
\hline Both & 4 & 24 \\
\hline
\end{tabular}

\section{ACKNOWLEDGEMENTS}

The authors respectfully acknowledge the National Science Foundation for Graduate Fellowship funding of Mr. Matthew Lee who participated on this project and the U.S. Department of Homeland Security for financial support of this project. The authors also acknowledge the U.S. Army Corps of Engineers Engineering Research and Development Center for their fruitful collaborations on this project, particularly their expert knowledge on levee systems and leadership in collection of ground truth data.

\section{REFERENCES}

[1] J. V. Aanstoos, K. Hasan, C. G. O’Hara, S. Prasad, L. Dabbiru, M. Mahrooghy, B. Gokaraju, M. Lee, R. Nobrega, "Earthen Levee Monitoring with Synthetic Aperture Radar", Proc. 2011 IEEE Applied Imagery Pattern Recognition Workshop, 2011.

[2] R. Gonzalez, and R. Woods, Digital Image Processing Second Edition, Upper Saddle River, N.J.: Prentice-Hall, Inc., 2002.

[3] Uninhabited Aerial Vehicle Synthetic Aperture Radar [Online].

http://www.jpl.nasa.gov/missions/index.cfm?mission=UAVSAR

[4] Climate of 2009 June in Historical Perspective [Online]. http://www.ncdc.noaa.gov/oa/climate/research/2009/jun/jun09.htm 1

[5] B. Sahiner, H.-P, Chan, N. Petrick, M. Helvie, and M. Goodsitt, "Computerized Characterization of Masses on Mammograms; The Rubber Band Straightening Transform and Texture Analysis,” Medical Physics, vol. 25, pp. 516 - 526, April 1998.

[6] Matthew A. Lee, "Analysis of Breast Lesions Using a Simplified Rubber Band Straightening Transform and the Onion Transform,” Master's Thesis, Mississippi State University, 2007. 